\title{
Geometric Metamorphosis
}

\author{
Marc Niethammer ${ }^{1,2}$, Gabriel L. Hart ${ }^{3}$, Danielle F. Pace ${ }^{3}$, Paul M. Vespa ${ }^{5}$, \\ Andrei Irimia ${ }^{4}$, John D. Van Horn ${ }^{4}$, and Stephen R. Aylward ${ }^{3}$ \\ 1 University of North Carolina (UNC), Chapel Hill NC 27599-3175, USA \\ 2 Biomedical Research Imaging Center, UNC Chapel Hill NC 27599-7515, USA \\ 3 Kitware, Inc., Carrboro NC 27510, USA \\ 4 Laboratory of Neuro Imaging, University of California, Los Angeles CA 90095, USA \\ 5 Brain Injury Research Center, University of California, Los Angeles CA 90095, USA
}

\begin{abstract}
Standard image registration methods do not account for changes in image appearance. Hence, metamorphosis approaches have been developed which jointly estimate a space deformation and a change in image appearance to construct a spatio-temporal trajectory smoothly transforming a source to a target image. For standard metamorphosis, geometric changes are not explicitly modeled. We propose a geometric metamorphosis formulation, which explains changes in image appearance by a global deformation, a deformation of a geometric model, and an image composition model. This work is motivated by the clinical challenge of predicting the long-term effects of traumatic brain injuries based on time-series images. This work is also applicable to the quantification of tumor progression (e.g., estimating its infiltrating and displacing components) and predicting chronic blood perfusion changes after stroke. We demonstrate the utility of the method using simulated data as well as scans from a clinical traumatic brain injury patient.
\end{abstract}

\section{Introduction and Background}

Image registration is based on structural similarity between a source and a target image. Similarity is often measured either by comparing image intensities directly or using indirect intensity measures like mutual information or cross correlation. However, for images with pathologies, assumptions of structural and intensity similarity may not hold.

In traumatic brain injury (TBI) cases, one clinical challenge is distinguishing permanent from transient changes in the brain in order to prescribe effective treatment and rehabilitation plans. Scans are acquired upon initial presentation in the clinic as well as after four to eight months (see Fig. 1). The geometry of the pathology, the deformation of the brain, and infiltration of the pathology into the brain change drastically between these scans. Determining the regions in which the infiltration has receded can be particularly useful in predicting longterm outcome. Similarly, in tumor cases, post-treatment assessment requires determination of changes in tumor geometry, tumor infiltration, scarring, and overall brain morphology. In stroke cases, there is a clinical need to predict chronic changes in blood perfusion from acute scans. In general, these cases are 
Fig. 1. MP-RAGE, post-contrast MRI scans from TBI case. Left: Initial scan. Right: Eight months after initial scan. Rigidly registered. $1 \mathrm{x} 1 \mathrm{x} 1 \mathrm{~mm}$ voxels.

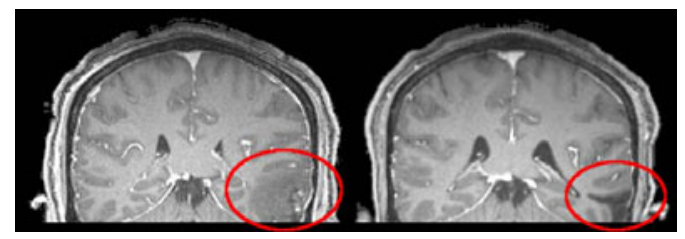

characterized by global tissue deformations, local changes in the geometry of a pathology, and local changes in the composition of the tissue and the pathology. We refer to these changes as "geometric metamorphosis."

While geometric metamorphosis changes may be tolerated by registration methods with low-dimensional image transformation models, direct application of a classical deformable registration method will likely produce unrealistic estimates of deformation. To address geometric metamorphosis changes, deformable registration approaches with weak and strong models of appearance change have been proposed. For example, methods having strong models of brain tumor mass effects and infiltration have been developed [47] and have been used to simulate tumors in atlas images to allow for spatial normalization of subjects with brain tumors [10. While highly sophisticated, these methods are application-specific and rely on a good match of the tumor model to the observed tumor. On the other hand, image metamorphosis methods [9] use weak models to smoothly transform a source to a target image exactly. However, the transformations estimated by image metamorphosis do not explicitly model the deformation or composition of the pathologies and instead compromise between a globally smooth spatial transformation and the interpolation of image intensities along individual point trajectories. Hence, image metamorphosis models will have difficulty quantifying effects such as tumor infiltration or tissue recovery in stroke. Consider also that the approach proposed in this paper is, in spirit, related to the methods proposed in [5]8|2] in which areas that cannot be matched (because no correspondence exists) are masked out. However, in those methods registration results inside these masked-out areas are only driven by the spatial regularity terms of the deformable registration algorithms. Our method explicitly includes a deformable geometric model of the extent of the appearance change in order to capture pathology deformations in conjunction with underlying image deformations.

Sec. 2 discusses the geometric metamorphosis model. Its numerical solution method is discussed in Sec. 3, Results are presented in Sec. 5. The paper concludes with a summary and outlook on future work in Sec. 6.

\section{Geometric Metamorphosis Model}

Taking tumor growth as an example, changes in image appearance can be caused by a mixture of tissue deformation, tumor growth displacing healthy tissue, and tumor infiltration into healthy tissue. Tissue deformation and displacement due to tumor growth could be captured (between time-points) using a standard 
registration method 1 . However, infiltration does not imply spatial changes. A registration method should be able to distinguish image appearance changes arising from the composition of background deformations of the image and foreground deformations of an embedded geometric object, e.g., a tumor.

We model these transformations through a fluid-registration formulation. In large displacement diffeomorphic metric mapping (LDDMM) 6] one minimizes

$$
E=\int_{0}^{1}\|v\|_{L}^{2} d t+\frac{1}{\sigma^{2}}\left\|I(1)-I_{1}\right\|^{2}, \quad \text { s.t. } \quad I_{t}+\nabla I^{T} v=0 ; I(0)=I_{0},
$$

where $v$ is a sought-for time-dependent velocity field which induces a spatial transformation warping the source image $I_{0}$ to the target image $I_{1}$. Typical LDDMM formulations register from source $I_{0}$ to target $I_{1}$ on the time interval $[0,1]$, thus $I(1)$ represents the warped source image. $L$ is a differential operator (here, $L=\gamma-\alpha \nabla^{2}, \alpha, \gamma>0$ ) controlling spatial regularity of $v ; \sigma>0$ controls the influence of the image match term. This is an inexact matching that only allows for the deformation of the source image $I_{0}$, but not for a change of its appearance. In order to explicitly model appearance changes, we augment this standard image registration model with an extra control to model foreground deformation of a geometric model (Sec. 2.1) which induces image changes through an image composition model (Sec. 2.2).

\subsection{Deformation Model}

Fig. 2 illustrates the principle of the geometric metamorphosis model. Since we model geometric metamorphosis as the composition of background and foreground deformations, we introduce the (smooth) indicator functions $T_{1}$ and $T_{2}$ as models of the geometric object, $T_{1}(x)$ and $T_{2}(x) \in[0,1]$. We then register the background global deformation on time $[0,1]$ and the foreground geometry change on time $(1,2]$, solving for the time-dependent velocity fields $v$ and $v^{\tau}$, respectively. We define the geometric metamorphosis problem as the minimization of

$$
\begin{aligned}
& E=(1-w) \int_{0}^{1}\|v\|_{L}^{2} d t+w \int_{1}^{2}\left\|v^{\tau}\right\|_{L}^{2} d t \\
& +\frac{1}{\sigma_{1}^{2}} \operatorname{Sim}\left(I^{c}\left(I_{1}, I^{\tau}(1), T_{2}\right), I^{c}\left(I(1), I^{\tau}(1), T_{2}\right)\right)+\frac{1}{\sigma_{2}^{2}} \operatorname{Sim}\left(I^{\tau}(2), T_{2}\right), \\
& \text { s.t. } I_{t}+\nabla I^{T} v=0 ; I(0)=I_{0}, \quad \begin{cases}I_{t}^{\tau}+\nabla\left(I^{\tau}\right)^{T} v=0, & I^{\tau}(0)=T_{1}, t \in[0,1], \\
I_{t}^{\tau}+\nabla\left(I^{\tau}\right)^{T} v^{\tau}=0, & t \in(1,2],\end{cases}
\end{aligned}
$$

where $I^{\tau}$ is the image of the geometric object and $w \in(0,1)$ controls the tradeoff between background and foreground deformations. Note that the geometric

\footnotetext{
${ }^{1}$ We assume for simplicity that the geometric object causing image change is present in both the source and target image, although it may undergo significant distortions.
} 
model $T_{1}$ and its image $I^{\tau}$ are subject to both deformations, whereas the source image is only subjected to the background deformation. $I^{c}(\cdot, \cdot, \cdot)$ denotes the image composition model (see Sec. 2.2). Sim denotes a similarity measure of choice. For simplicity, we use the $L^{2}$ distance measure, $\operatorname{Sim}(I, J)=\|I-J\|^{2}$. Two similarity terms are used to assure matching of (i) the regions which correspond in both images and (ii) the geometric models.

\subsection{Image Composition Model}

To accommodate local expansions and contractions of the geometric model affecting image appearance, the image composition model needs to preserve regions where the source and target image can be reliably matched. It needs to disregard areas where no matching image information can be found due to the shape change of the geometric model. The composition model

$$
I^{c}\left(I, I^{\tau}(1), T_{2}\right)(x):=I(x)\left(1-I^{\tau}(1, x)\right)\left(1-T_{2}(x)\right),
$$

achieves this by zeroing out regions defined by the smoothed indicator functions $I^{\tau}(1)$ and $T_{2}$. Since this happens for both arguments of the similarity function in Eq.1 the image match is effectively disregarded in these regions. This definition is reminiscent of cost function masking as for example used when registering images with and without lesions [2]. Here, we use regions in the source and target image to alter the energy function and estimate the regions which should be excluded in a joint optimization process. This allows for a combined estimation of foreground and background deformation.

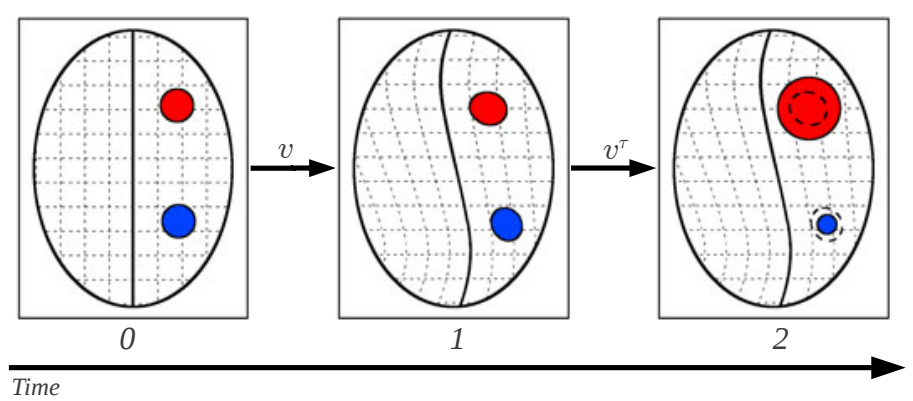

Fig. 2. Geometric Metamorphosis. An image is explained by a global deformation (via $v$ ) and a geometric model deformation (via $v^{\tau}$ ). Corresponding structures in the source and target guide the estimation of $v$ and $v^{\tau}$ addresses additional appearance differences at the pathology. To avoid faulty evaluation of image similarities, a suitable image composition method is required (Sec. 2.2). Regions which carry no matchable information are set to 0 in the image composition model. For a shrinking geometric model (blue) this region is specified by $I^{\tau}(1)$ (which already includes the background deformation) and for a growing geometric model (red) by $T_{2}$. Defining the composition model as Eq. 2 allows localized growing and shrinking simultaneously. 


\section{$3 \quad$ Numerical Solution}

We follow the solution method of 3 to solve the registration problem. To compute the optimality conditions, we add the dynamic constraints through the Lagrange multipliers $\lambda$ and $\lambda^{\tau}$. Note that $\lambda^{\tau}$ is allowed to be discontinuous at $t=1$ due to the energy term depending on $I^{\tau}(1)$. After some computations we obtain the optimality conditions for $t \in[0,1)$

$$
\begin{aligned}
0 & =2(1-w) L^{\dagger} L v+\lambda \nabla I+\lambda^{\tau} \nabla I^{\tau} \\
I_{t}+\nabla I^{T} v & =0, I(0)=I_{0} \\
-\lambda_{t}-\operatorname{div}(\lambda v) & =0, \lambda(1)=\frac{2}{\sigma_{1}^{2}}\left(I_{1}-I(1)\right)\left(1-T_{2}\right)^{2}\left(1-I^{\tau}(1)\right)^{2} \\
I_{t}^{\tau}+\nabla\left(I^{\tau}\right)^{T} v & =0, I^{\tau}(0)=T_{1} \\
-\lambda_{t}^{\tau}-\operatorname{div}\left(\lambda^{\tau} v\right) & =0, \lambda^{\tau}(1-)=\lambda^{\tau}(1+)+\frac{2}{\sigma_{1}^{2}}\left(I(1)-I_{1}\right)^{2}\left(1-T_{2}\right)^{2}\left(1-I^{\tau}(1)\right),
\end{aligned}
$$

and for $t \in(1,2]$

$$
\begin{aligned}
2 w L^{\dagger} L v^{\tau}+\lambda^{\tau} \nabla I^{\tau} & =0, \\
I_{t}^{\tau}+\nabla\left(I^{\tau}\right)^{T} v^{\tau} & =0, \\
-\lambda_{t}^{\tau}-\operatorname{div}\left(\lambda^{\tau} v^{\tau}\right) & =0, \lambda^{\tau}(2)=-\frac{2}{\sigma_{2}^{2}}\left(I^{\tau}(2)-T_{2}\right) .
\end{aligned}
$$

The final conditions for $\lambda$ and $\lambda^{\tau}$ in $[0,1)$ reflect the "don't care" areas of the registration: areas where $T_{2}=1$ or $I^{\tau}(1)=1$ are zeroed out. This is sensible, because the Lagrangian multipliers represent the image-matching error. We obtain a solution fulfilling the optimality conditions through the following adjoint solution method:

0) Initialize $v, v^{\tau}$ to zero.

1) Solve $I_{t}+\nabla I^{T} v=0, I(0)=I_{0}$ and $I_{t}^{\tau}+\nabla\left(I^{\tau}\right)^{T} v=0, I^{\tau}(0)=T_{1}$ forward in time in $[0,1]$.

2) Continue solving for $I^{\tau}$ for $t \in(1,2]$ but with velocity field $v^{\tau}$.

3) Compute the adjoint solution $\lambda$ backward for $t \in[0,1]$.

4) Compute the adjoint solution $\lambda^{\tau}$ backward for $t \in(1,2]$.

5) Apply the jump condition to $\lambda^{\tau}$ at $t=1$.

6) Compute the adjoint solution $\lambda^{\tau}$ backward for $t \in[0,1)$.

5) Compute for every point and time-point the gradients

$$
\begin{array}{r}
\nabla_{v}(t) E=2(1-w) L^{\dagger} L v+\lambda \nabla I+\lambda^{\tau} \nabla I^{\tau}, t \in[0,1], \\
\nabla_{v^{\tau}}(t) E=2 w L^{\dagger} L v^{\tau}+\lambda^{\tau} \nabla I^{\tau}, t \in(1,2] .
\end{array}
$$

6) Do a gradient descent step to update the velocities (using line search).

7) Repeat steps 1 - 6 until convergence. 


\section{Estimating Geometric Deformation}

Once the foreground and background velocity fields $v$ and $v^{\tau}$ have been estimated, they can be used to represent the geometric deformation modulo the background deformation. This allows for visualization and quantification, for example of tumor growth. Computing (backward in time) the mapping

$$
-\Phi_{t}^{r}-D \Phi^{r} v=0, \Phi^{r}(1)=i d, t \in[0,1]
$$

where $i d$ is the identity map and $D$ the Jacobian, shape change is computed as

$$
S(0)=T_{2} \circ \Phi^{r}(0)-T_{1}, \quad S(1)=T_{2}-I^{\tau}(1)
$$

in the coordinate system of the source and the target image respectively. Here positive values indicate expansion and negative values contraction with respect to the source image.

\section{$5 \quad$ Experimental Results}

We test the geometric metamorphosis model on two sets of synthetic images, and a TBI image pair. The first synthetic set (Fig. 3) illustrates four different scenarios: (i) all change caused by infiltration, (ii) all change caused by global deformation, (iii) global deformation and local infiltration, and (iv) global deformation and local recession.

The second synthetic set consists of ten different global and object warps applied to the same source image and geometric object. After registering, we compute the mean and standard deviation of the percent overlap for the geometric object as,

$$
\operatorname{Overlap}\left(T_{2}, I^{\tau}(2)\right)=\operatorname{sum}\left(\left(I_{\geq 0.5}^{\tau}(2)\right) \cap\left(T_{2, \geq 0.5}\right)\right) / \operatorname{sum}\left(\left(T_{2, \geq 0.5}\right)\right),
$$

where $I_{\geq x}$ is a binary mask of all pixels in $I$ greater than or equal to $x$.

We also compute the background registration accuracy using six manually selected landmarks in the tissue region as ground truth. Landmark locations are calculated on the pixel grid and are accurate to $+/-0.5$ pixels. We compare the results of our method against the B-Spline and LDDMM registration methods. Given the expected similar results in the regions of the image away from the geometric object we look at the extreme percentiles of the landmark distance mismatch values. Both LDDMM and geometric metamorphosis compute $95 \%$ of the landmarks to within 0.5 pixels of their correct location, but our method is able to achieve a significantly higher overlap accuracy (Fig. 3).

The TBI test case contains considerable deformation as well as object recession around the pathology site. To illustrate those changes, we provide manual segmentations of the pathology sites in both images (Fig. 4) $]^{2}$. Of clinical importance, Fig. [5 shows the progression of the pathology label map over the entire time solution interval $[0,2]$.

\footnotetext{
${ }^{2}$ Note that it is expected that a high segmentations accuracy is not required [1].
} 


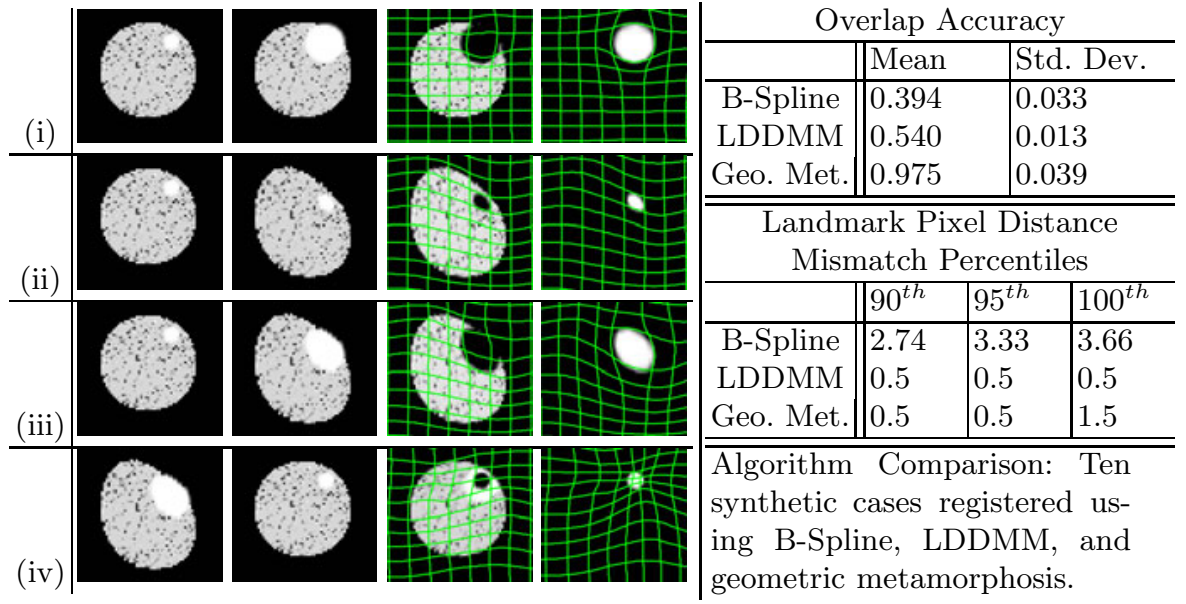

Fig. 3. Synthetic Results. Each row, left to right: $I_{0}, I_{1}, I(1)$ and global deformation, $I^{\tau}(2)$ and composite deformation. (i) Local infiltration. (ii) Image deformation. (iii) Image deformation and local infiltration. (iv) Image deformation and local recession.

(a)

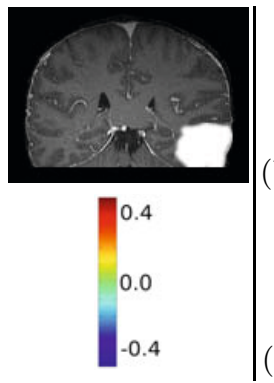

(b)

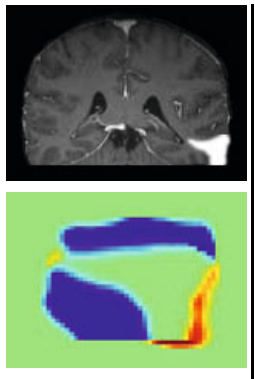

(c)
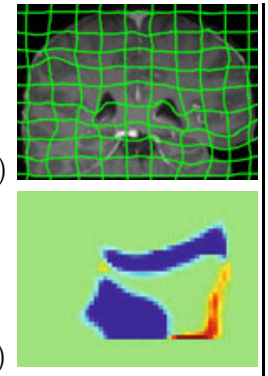

(d)
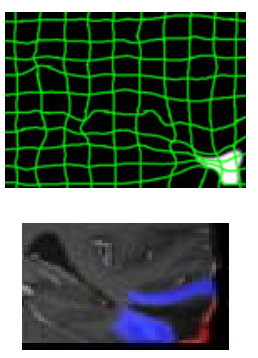

Fig. 4. TBI Results. Top: (a) Initial scan, $T_{1}$ overlaid. (b) Second scan, $T_{2}$ overlaid. (c) Image deformation and $I(1)$. (d) Retraction area deformation and $I^{\tau}(2)$. Bottom: (e) $S(0)$ : Shape change in the source image coordinate frame (f) $S(1)$ : Shape change in the target image coordinate frame, (g) Incursion (red) and retraction (blue) in $I_{2}$.

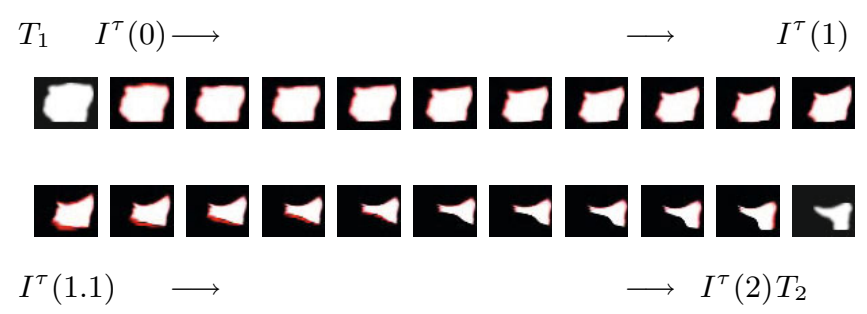

Fig. 5. TBI Pathology Label Map. Progression of TBI pathology label map on $[0,2]$. Red portion of each frame shows the change from the previous time point. Top: $[0,1]$ Changes in global deformation. Bottom: $(1,2]$ Changes in infiltration and recession. 


\section{Conclusions and Future Work}

We proposed an image registration method allowing for background deformation of a source image, foreground deformation of a geometric object, and their composition to match a target image. The method can thereby account for processes causing images to change due to pathology infiltration/recession and image deformation. Since it makes minimal assumptions about the underlying change, it is generally applicable. We demonstrated its behavior for the registration of simulated data and traumatic brain injury cases. If desired, the registration framework can be augmented with an application-specific model, for example, of tumor growth, as in 4. In future work we will investigate adaptations wherein a geometric model is only available in one of the two images and a transformed model either needs to be estimated from the image or does not exist, e.g., when registering a tumor patient to a healthy atlas. This work was sponsored in part by the following grants: NIH 1R01CA138419-01, NIH 1U54EB005149-01, NIH 1R01MH091645-01A1, NIH 2P41EB002025-26A1 and NSF EECS-0925875.

\section{References}

1. Anderson, S.M., Rapcsak, S.Z., Beeson, P.M.: Cost function masking during normalization of brains with focal lesions: still a necessity? Neuroimage 53(1), 78-84 (2010)

2. Brett, M., Leff, A., Rorden, C., Ashburner, J.: Spatial normalization of brain images with focal lesions using cost function masking. Neuroimage 14(2), 486-500 (2001)

3. Hart, G.L., Zach, C., Niethammer, M.: An optimal control approach for deformable registration. In: MMBIA Workshop, CVPR (2009)

4. Hogea, C., Davatzikos, C., Biros, G.: An image-driven parameter estimation problem for a reaction-diffusion glioma growth model with mass effects. Journal of Mathematical Biology 56(6), 793-825 (2008)

5. Lamecker, H., Pennec, X.: Atlas to image-with-tumor registration based on demons and deformation inpainting. In: Proc. MICCAI Workshop on Computational Imaging Biomarkers for Tumors - From Qualitative to Quantitative, CIBT 2010 (2010)

6. Miller, M.I., Younes, L.: Group actions, homeomorphisms, and matching: A general framework. International Journal of Computer Vision 41(1/2), 61-84 (2001)

7. Prastawa, M., Bullitt, E., Gerig, G.: Simulation of brain tumors in MR images for evaluation of segmentation efficacy. Medical Image Analysis 13(2), 297-311 (2009)

8. Stefanescu, R., Commowick, O., Malandain, G., Bondiau, P., Ayache, N., Pennec, $\mathrm{X}$.: Non-rigid atlas to subject registration with pathologies for conformal brain radiotherapy. In: Barillot, C., Haynor, D.R., Hellier, P. (eds.) MICCAI 2004. LNCS, vol. 3216, pp. 704-711. Springer, Heidelberg (2004)

9. Trouve, A., Younes, L.: Metamorphoses through Lie group action. In: Foundations of Computational Mathematics, pp. 173-198 (2005)

10. Zacharaki, E., Hogea, C., Shen, D., Biros, G., Davatzikos, C.: Non-diffeomorphic registration of brain tumor images by simulating tissue loss and tumor growth. Neuroimage 46(3), 762-774 (2009) 